Journal Club

Editor's Note: These short, critical reviews of recent papers in the Journal, written exclusively by graduate students or postdoctoral fellows, are intended to summarize the important findings of the paper and provide additional insight and commentary. For more information on the format and purpose of the Journal Club, please see http://www.jneurosci.org/misc/ifa_features.shtml.

\title{
Targeting Axonal Regeneration: The Growth Cone Takes the Lead
}

\author{
Laura Montani ${ }^{1 *}$ and Marija M. Petrinovic ${ }^{2,3 *}$ \\ ${ }^{1}$ Institute of Molecular Health Sciences, Swiss Federal Institute of Technology, ETH, Zurich, CH-8093, Zurich, Switzerland, ${ }^{2}$ F. Hoffmann-La Roche, pRED, \\ Pharma Research and Early Development, DTA Neuroscience, Ophthalmology and Rare Diseases, CH-4070 Basel, Switzerland, and ${ }^{3}$ Biozentrum, University \\ of Basel, CH-4056, Basel, Switzerland \\ Review of Stern et al.
}

Understanding the mechanisms that mediate axonal regeneration and functional recovery after nervous system injury has been a long-lasting goal of neuroscience. In marked contrast to the adult central nervous system (CNS), the peripheral nervous system (PNS) is "regeneration friendly." Whereas CNS neurons form short, local sprouts and achieve only partial recovery by reprogramming uninjured pathways, PNS axons mount a robust regenerative response and reinnervate their targets after a lesion, thereby producing substantial functional recovery (Huebner and Strittmatter, 2009). These contrasting regenerative capacities have been attributed in part to the presence of growth-supportive Schwann cells in the PNS and a growth-inhibiting environment produced by oligodendrocytes and glial scar in the CNS (Huebner and Stritt-

Received Jan. 23, 2014; revised Feb. 17, 2014; accepted Feb. 20, 2014. M.M.P. is supported by a Roche Postdoctoral Fellowship (RPF) and L.M. by a Swiss National Foundation Marie Heim-Vögtlin Fellowship (PMPDP3 139610). We thank our mentors Dr. Basil Künnecke, Prof. Peter Scheiffele, and Prof. Ueli Suter for their supervision, insightful discussions, and helpful comments on this manuscript.

*L.M. and M.M.P. contributed equally to this work.

Correspondence should be addressed to Marija M. Petrinovic, F. Hoffmann-La Roche, pRED, Pharma Research and Early Development, DTA Neuroscience, Ophthalmology and Rare Diseases, Building 68, Room 327, Grenzacherstrasse 124, CH-4070, Basel, Switzerland, E-mail: marija-magdalena.petrinovic@roche.com; or Laura Montani, Institute of Molecular Health Sciences, Swiss Federal Institute of Technology, ETH, Zurich, Schafmattstrasse 22, CH-8093, Zurich, Switzerland, E-mail: laura.montani@biol.ethz.ch.

DOI:10.1523/JNEUROSCI.0320-14.2014

Copyright $\odot 2014$ the authors $\quad 0270-6474 / 14 / 344443-02 \$ 15.00 / 0$ matter, 2009). The inhibitory environment of the CNS, in combination with low intrinsic growth properties of CNS axons, results in collapse of axonal growth cones and axon retraction (Huebner and Strittmatter, 2009). In contrast, the PNS axons assemble new actin-rich growth cones soon after injury (Bradke et al., 2012). Growth cone assembly, motility, and navigation in response to extracellular signals are regulated by actin dynamics (Bradke et al., 2012), but until recently, little was known about the molecular mechanisms that control growth cone dynamics after a PNS lesion.

An important piece of this puzzle was recently provided by Stern and colleagues (2013), who identified the transcription factor serum response factor (SRF) as an important reactivator of growth cone dynamics during PNS axonal regeneration. SRF is a versatile transcription factor previously shown to have roles in regulating growth cone actin dynamics in cultured neurons and in neuronal migration, neurite outgrowth, pathfinding, and synaptic targeting during development (Knöll and Nordheim, 2009), presumably by regulating gene transcription. Furthermore, by activating genes involved in cytoskeleton remodeling, SRF plays an instrumental role in converting synaptic activity into plasticity-associated structural changes: a fundamental process for regeneration and functional recovery following nervous system injury (Knöll and Nordheim, 2009).

Stern and colleagues (2013) showed that virus-mediated genetic depletion of SRF in facial motoneurons resulted in decreased regeneration and axonal sprouting of transected facial nerves. This finding is consistent with previous observations that SRF deletion causes deficits in neurite outgrowth, guidance, and migration (Knöll and Nordheim, 2009). Astonishingly, however, the role of SRF in supporting PNS regeneration seemed not to involve enhancement of transcription, but rather to require translocation from the nucleus to the cytoplasm. Stern and colleagues (2013) demonstrated this new function of SRF by severing the facial nerve in mice expressing not only endogenous SRF but also overexpressing a mutant SRF form that lacks the nuclear localization signal and therefore remains confined in the cytoplasm. They observed highly increased regeneration and axonal sprouting, suggesting that SRF promotes regeneration upon entering the cytoplasm. In addition, as was previously shown when wild-type SRF was overexpressed, viral-mediated overexpression of cytoplasmic SRF reduced neuronal loss, suggesting a possible neuroprotective effect (Stern et al., 2012, 2013). Intriguingly, cytoplasmically overexpressed SRF also enhanced regenerative capacity of neighboring, nontransfected cells by mechanisms that 
have not yet been fully elucidated (Stern et al., 2013).

Led by the observation that both nuclear and cytoplasmic SRF show neuroprotective effects, but that only cytoplasmically localized SRF affects neurite outgrowth, Stern and colleagues (2013) examined the functional consequences of cytoplasmic SRF on neurite growth and growth cone morphology. To this end, they overexpressed cytoplasmically targeted SRF in cultured primary CNS and PNS neurons. In addition to expanding the number of neurons, cytoplasmically overexpressed SRF increased the length of neurites, amount of F-actin, number of filopodia, and area of growth cones, suggesting that cytoplasmic SRF may directly affect actin-driven cytoskeletal dynamics. Conversely, nuclear SRF controlled cytoskeletal dynamics through transcriptional modulation of several cytoskeletal genes and actin binding proteins (Knöll and Nordheim, 2009). Indeed, Stern and colleagues (2013) showed that upregulation of cytoplasmic SRF led to dephosphorylation of the actin-severing protein cofilin, likely resulting in its activation and in increased actin dynamics. Interestingly, after facial nerve injury, phosphorylated, i.e., inactive, cofilin was found to translocate from the cytoplasm to the nucleus (Stern et al., 2013). This translocation was strongly impaired in facial motoneurons overexpressing cytoplasmic SRF (Stern et al., 2013), suggesting that by promoting cofilin dephosphorylation, SRF blocks its nuclear translocation. However, SRF is unlikely to be directly involved in mediating this process and thus the phosphatase responsible for cofilin dephosphorylation upon SRF cytoplasmic translocation remains to be ascertained. The reverse was also true, i.e., cofilin regulated cytoplasmic levels of phosphorylated SRF (Stern et al., 2013). These data suggest that a regulatory loop consisting of SRF and cofilin might impart reactivation of actin dynamics in growth-inert retraction bulbs and facilitate axonal regeneration.

This study describes SRF as an axonal growth promoting molecule that, surprisingly, exerts its function by translocating from the nucleus toward the axonal/ growth cone compartment. There it stimulates intracellular signaling pathways that converge on rearranging the actin cytoskeleton by signaling to cofilin. How cytoplasmic SRF indirectly enhances cofilin dephosphorylation is unclear, but clues might be gleaned by examining the mechanisms of growth restriction by myelinassociated inhibitory proteins. These promote growth cone collapse and axon retraction through activation of the small GTPase RhoA, causing the downstream modulation of cofilin activity (Hsieh et al., 2006; Montani et al., 2009). Interestingly, RhoA activity is also increased in regeneration "non-friendly" SRF mutants (Knöll and Nordheim, 2009). Furthermore, RhoGTPases require SRF activity to fulfil their function in growth cone guidance, as shown by the insensitivity of SRF mutant neurites to guidance molecules (Knöll and Nordheim, 2009). Importantly, peripheral nerves can regenerate to a large extent following a lesion, despite the presence of the myelin-associated glycoprotein, one of the most studied myelin-associated inhibitory proteins (Shen et al., 1998). These findings, combined with those recently reported by Stern and colleagues (2013), suggest that cytoplasmic SRF on its own is sufficient to counteract activation of Rho-GTPases and restore growth cone dynamics and neurite outgrowth even in the presence of myelin-associated inhibitory proteins. Cytoplasmic SRF might therefore be able to promote growth of CNS axons after injury. However, the experimental evidence presented in the study of Stern and colleagues (2013) suggests that cytoplasmic SRF enhances rather shortrange axonal growth and sprouting of cultured CNS, as well as PNS neurons in vivo. This would suggest that cytoplasmic SRF in the CNS may be a target for increasing postlesion plasticity, more than long distance axonal regeneration. Furthermore, it is important to note here that regeneration per se is not a synonym for functional recovery, and that one of the most important determinants of a favorable functional outcome is the accuracy of target reinnervation. This aspect was not examined in the study by Stern and colleagues (2013), although it was previously shown that SRF plays important roles in axonal guidance and synapse formation (Knöll and Nordheim, 2009).

In summary, Stern and colleagues (2013) have unmasked nucleus-to-cytoplasm shuttling and subsequent indirect cofilin dephosphorylation as a mechanism by which SRF stimulates axonal regeneration in the injured nervous system. With this, they introduced a further level of com- plexity to our current understanding of the signaling pathways involved in axonal regeneration. Identification of cytoplasmic SRF as a modulator of growth cone dynamics during axonal regeneration in the PNS represents an important step toward the development of new potential treatments to improve CNS regeneration. However, a note of caution applies to SRF as a potential pharmacological target: it has been shown to enhance amyloid- $\beta$ accumulation in vessels and to accelerate experimental tumor metastasis (Knöll and Nordheim, 2009). Although further research is needed to clarify the molecular mechanisms responsible for SRF nucleusto-cytoplasm translocation and signaling, as well as for elucidation of potential translatability to the CNS, this study by Stern and colleagues (2013) holds promise to open up a new avenue for enticing regeneration of the nervous system after injury.

\section{References}

Bradke F, Fawcett JW, Spira ME (2012) Assembly of a new growth cone after axotomy: the precursor to axon regeneration. Nat Rev Neurosci 13:183-193. CrossRef Medline

HsiehSH, Ferraro GB, FournierAE (2006) Myelinassociated inhibitors regulate cofilin phosphorylation and neuronal inhibition through LIM kinase and slingshot phosphatase. J Neurosci 26: 1006-1015. CrossRef Medline

Huebner EA, Strittmatter SM (2009) Axon regeneration in the peripheral and central nervous systems. Results Probl Cell Differ 48: 339-351. CrossRef Medline

Knöll B, Nordheim A (2009) Functional versatility of transcription factors in the nervous system: the SRF paradigm. Trends Neurosci 32:432-442. CrossRef Medline

Montani L, Gerrits B, Gehrig P, Kempf A, Dimou L, Wollscheid B, Schwab ME (2009) Neuronal Nogo-A modulates growth cone motility via Rho-GTP/LIMK1/cofilin in the unlesioned adult nervous system. J Biol Chem 284: 10793-10807. CrossRef Medline

Shen YJ, DeBellard ME, Salzer JL, Roder J, Filbin MT (1998) Myelin-associated glycoprotein in myelin and expressed by Schwann cells inhibits axonal regeneration and branching. Mol Cell Neurosci 12:79-91. CrossRef Medline

Stern S, Sinske D, Knöll B (2012) Serum response factor modulates neuron survival during peripheral axon injury. J Neuroinflammation 9:78. CrossRef Medline

Stern S, Haverkamp S, Sinske D, Tedeschi A, Naumann U, Di Giovanni S, Kochanek S, Nordheim A, Knöll B (2013) The transcription factor serum response factor stimulates axon regeneration through cytoplasmic localization and cofilin interaction. J Neurosci 33: 18836-18848. CrossRef Medline 